\title{
How Primary Care Providers Talk to Patients About Alcohol
}

\section{A Qualitative Study}

Kinsey A. McCormick, BA, 1,2 Nancy E. Cochran, MD, ${ }^{3,4}$ Anthony L. Back, MD, ${ }^{2}$ Joseph O. Merrill, MD, MPH, ${ }^{2,5}$ Emily C. Williams, $M P H^{2,6}$ Katharine A. Bradley, MD, MPH, $1,6,7,8$

${ }^{1}$ Northwest Health Services Research and Development Center of Excellence, VA Puget Sound Health Care System, Seattle, WA, USA; ${ }^{2}$ Department of Medicine, University of Washington, Seattle, WA, USA; ${ }^{3}$ White River Junction VA Hospital, White River Junction, VT, USA; ${ }^{4}$ Department of Medicine and Community and Family Medicine, Dartmouth Medical School, Hanover, $\mathrm{NH}$, USA; ${ }^{5} \mathrm{Harborview}$ Medical Center, Seattle, WA, USA; ${ }^{6}$ Center of Excellence in Substance Abuse Treatment and Education, VA Puget Sound Health Care System, Seattle, WA, USA; ${ }^{7}$ Primary and Specialty Medical Care Service, VA Puget Sound Health Care System, Seattle, WA, USA; ${ }^{8}$ Department of Health Services, University of Washington, Seattle, WA, USA.

BACKGROUND: Alcohol misuse is a common and well-documented source of morbidity and mortality. Brief primary care alcohol counseling has been shown to benefit patients with alcohol misuse.

OBJECTIVE: To describe alcohol-related discussions between primary care providers and patients who screened positive for alcohol misuse.

DESIGN: An exploratory, qualitative analysis of audiotaped primary care visits containing discussions of alcohol use.

PARTICIPANTS: Participants were 29 male outpatients at a Veterans Affairs (VA) General Internal Medicine Clinic who screened positive for alcohol misuse and their 14 primary care providers, all of whom were participating in a larger quality improvement trial.

MEASUREMENTS: Audiotaped visits with any alcohol-related discussion were transcribed and coded using grounded theory and conversation analysis, both qualitative research techniques.

RESULTS: Three themes were identified: (1) patients disclosed information regarding their alcohol use, but providers often did not explore these disclosures; (2) advice about alcohol use was typically vague and/or tentative in contrast to smoking-related advice, which was more common and usually more clear and firm; and (3) discomfort on the part of the provider was evident during alcohol-related discussions.

LIMITATIONS: Generalizability of findings from this single-site VA study is unknown.

CONCLUSION: Findings from this single site study suggest that provider discomfort and avoidance are important barriers to evidencebased brief alcohol counseling. Further investigation into current alcohol counseling practices is needed to determine whether these patterns extend to other primary care settings, and to inform future educational efforts.

KEY WORDS: alcohol drinking; primary care; communication; physician-patient relations.

DOI: $10.1111 / \mathrm{j} .1525-1497.2006 .00490 . x$

J GEN INTERN MED 2006; 21:966-972.

A lcohol misuse is common and is an important source of preventable morbidity and mortality. ${ }^{1,2}$ Based on trials showing that brief alcohol counseling reduces alcohol consumption, ${ }^{3-6}$ adverse health consequences, ${ }^{7}$ and health care

None of the authors have any conflicts of interest to declare.

A preliminary version of this study was presented at the Society of General Internal Medicine annual meeting, Chicago, IL, May 14, 2004.

Address correspondence and requests for reprints to Dr. McCormick: VA Puget Sound Health Services Research and Development, (152) 1100 Olive Way, Suite 1400, Seattle, WA 98101 (e-mail: kamc@u.washington. edu). utilization ${ }^{8-10}$ in patients who drink above recommended limits, the U.S. Preventive Services Task Force recommends routine alcohol screening, followed by brief counseling with patients who screen positive. ${ }^{2}$ Effective brief alcohol counseling is typically patient-centered ${ }^{11}$ and includes expression of concern, feedback linking the patient's drinking and health, explicit advice to reduce drinking, and agreement on a patientoriented plan. ${ }^{2,12}$

Providers generally believe that addressing alcohol misuse is an important clinical responsibility. ${ }^{13}$ However, rates of alcohol counseling are low, ${ }^{14-16}$ ambitious efforts to implement primary care alcohol counseling have not succeeded, ${ }^{17,18}$ and providers report a number of barriers to having alcoholrelated discussions. Such barriers include confusion as to what constitutes alcohol misuse, ${ }^{13}$ fear that asking about drinking could harm the patient-provider relationship, ${ }^{19-21}$ stigmatization of substance abuse, ${ }^{13,22}$ skepticism about the effectiveness of alcohol counseling, ${ }^{13,21,23}$ lack of time, ${ }^{19-21,24-26}$ inadequate training, ${ }^{13,27}$ and a belief that patients will not honestly disclose their drinking practices. ${ }^{20,21,28}$ Little is known, however, about how providers actually talk to patients about alcohol use, as most studies have relied on patient or provider self-report.

The purpose of this exploratory study was to describe audiotaped alcohol-related discussions between patients who screened positive for alcohol misuse and their primary care providers. Using a qualitative approach, we sought to identify major themes that emerged during discussions of alcohol use in a primary care setting.

\section{METHODS}

This single-site study was designed as a companion to the multisite Ambulatory Care Quality Improvement Project (ACQUIP). ${ }^{29}$ The ACQUIP trial used mailed surveys to identify patients with 6 conditions, including alcohol misuse, and evaluated whether giving providers information about their patients' self-reported health status before each visit improved patient satisfaction and health status. The Ambulatory Care Quality Improvement Project was conducted at 7 VA General Internal Medicine (GIM) clinics, and all GIM patients with a visit in the past year were eligible. At each site, GIM providers were assigned to either the Intervention or Usual Care firm, with Intervention providers receiving feedback about their

Manuscript received August 9, 2005

Initial editorial decision October 4, 2005

Final acceptance March 24, 2006 
patients' health status. The ACQUIP intervention had no significant beneficial effect. ${ }^{29}$

\section{Data Collection}

Audiotapes for the present study were collected at 1 ACQUIP site. Providers were invited to participate in a study of patientprovider communication by an investigator whose research does not relate to alcohol. All GIM providers at the study site were eligible, except 4 , who were involved with design or institutional approval of this audiotape study ( $n=84$ eligible).

Patients of consenting providers were eligible if they screened positive for alcohol misuse on the baseline ACQUIP survey and indicated on a separate mailed form that they would be willing to consider participating in an audiotape study of patient-provider communication. Patients screened positive if they reported any alcohol use in the past year and reported drinking above recommended limits ( $\geq 14$ drinks per week or 5 or more drinks per occasion), ${ }^{30}$ scored $\geq 1$ point on the CAGE questionnaire (standard timeframe of "ever"), or reported ever having had a drinking problem. ${ }^{31}$ This validated screening strategy has $92 \%$ sensitivity and $50 \%$ specificity for the detection of alcohol misuse in a male, VA primary care population, ${ }^{31}$ and a similar strategy has been used previously in trials of brief alcohol counseling. ${ }^{7,8}$ A cut-point $\geq 1$ was used because the standard CAGE cut-point $(\geq 2)$ has low sensitivity (53\%) for alcohol misuse in this population. ${ }^{32}$ of 7,700 patients from the study clinic who were eligible for ACQUIP, $4,046(52 \%)$ returned the baseline survey before the start of the audiotape study, and 840 (21\%) screened positive for alcohol misuse. Of those, 279 indicated that they were willing to consider participating in an audiotape study of patient-provider communication, and 130 had a consenting provider. ${ }^{33}$ Female patients were not audiotaped because of inadequate numbers. ${ }^{33}$

Before the first visit between eligible patient-provider pairs, a research assistant (RA) obtained written informed consent from the patient, including explicit consent to allow review of the audiotapes by unnamed investigators. For this and subsequent visits, the RA placed a tape recorder in the provider's room and retrieved the audiotape after each appointment. The audiotapes were transcribed with identifying details deleted.

The previously published quantitative analysis of these audiotapes described the frequency of prespecified patient and provider behaviors during the alcohol-related discussions. ${ }^{33}$ During coding for the previous study, investigators noted qualitative aspects of patient-provider interactions not captured by the quantitative coding system. Two investigators with expertise in qualitative research methodology and 2 new coders were recruited to conduct qualitative analyses. The study was approved by the local institutional review board.

At the end of the study, patients and providers completed a survey, which evaluated whether they believed that the study was focused on any of 9 conditions, including alcohol misuse. No participant believed that alcohol misuse was the study's sole focus. ${ }^{33}$

Among eligible patients with a consenting provider ( $n=130$ ), 98 had 1 or more appointments during the 6-month study period, 53 consented, and 47 were audiotaped at least once (Fig. 1). Of 68 visits recorded, 39 contained some reference to alcohol and were included in analyses presented here. $^{33}$

\section{Coding and Analytic Methods}

Two authors (K.M., N.C.) independently listened to and coded the 39 visits in their entirety. Coders did not discern systematic, qualitative differences between Intervention and Usual Care providers or between types of providers, so audiotapes were analyzed as a single data set.

A descriptive thematic analysis of the audiotapes, an approach based on grounded theory, ${ }^{34}$ was used. In particular, attention was paid to the sequence of, timing, and responses to verbal statements. ${ }^{35,36}$ The 2 coders performed open-coding of all 39 tapes, identifying patient and provider behaviors as they emerged from the data. The lead author grouped excerpts illustrating these behaviors into categories, with some excerpts falling under multiple categories, and then organized the categories into broader thematic groups. Categories were intended to be neutral and purely descriptive-for instance, "Provider changes subject" and "Patient initiates alcohol discussion." To maximize trustworthiness (a concept comparable to internal validity for quantitative studies), all authors reviewed and critiqued 3 interim versions of the themes, proposing alternative interpretations and asking for further supporting observations for each theme. This iterative process resulted in progressive refinement of the themes.

To illustrate each theme, 3 to 4 examples were chosen based on their clarity and interpretability from a brief excerpt.

\section{RESULTS}

\section{Study Population}

Of 84 eligible providers, 34 (40\%) consented to participate, and 17 had a visit with an eligible patient during the study period and were audiotaped. ${ }^{33}$ Taped providers were more likely than nontaped providers to be staff physicians $(65 \%$ vs $35 \%$, $P=$.026), but no gender differences were found. ${ }^{33}$ Of the 14 providers who discussed alcohol use with 1 or more patients (Table 1), 5 practiced in the ACQUIP Intervention firm and received reports about their patients' drinking.

Of 47 taped patients, 29 discussed alcohol use with their provider (Table 1) in a total of 39 visits. Taped patients had a mean age of 61 , and a higher incidence of chronic obstructive pulmonary disease compared with nontaped patients $(n=793)$ (38\% vs $25 \%, P=.05){ }^{33}$ Twenty-seven patients reported pastyear binge drinking and 17 screened positive on the standard CAGE.

\section{Themes}

Three themes emerged from the 39 visits in which alcohol was discussed. Alcohol-related discussions lasted from 2 seconds to 7 minutes, 17 seconds (median duration 45 seconds; interquartile range 26 seconds to 2 minutes, 40 seconds).

Patients Disclosed Information Regarding Their Alcohol Use, But Providers Often Did Not Explore These Disclosures. We observed many instances of patients' disclosing potentially important information regarding their drinking, both in response to provider's questioning and without any prompting. Specifically, patients disclosed high levels of consumption and negative health consequences. Providers, however, often failed to pursue these disclosures.

Providers responded to patient disclosures in several ways. Some abruptly changed the subject (Table 2; Examples 1, 2, 3), 


\section{Screened Positive for Alcohol Misuse} \& were Surveyed:

"Would you be willing to consider participating in a study of patient-provider communication by allowing all your GIMC visits to be tape recorded for up to a year?"

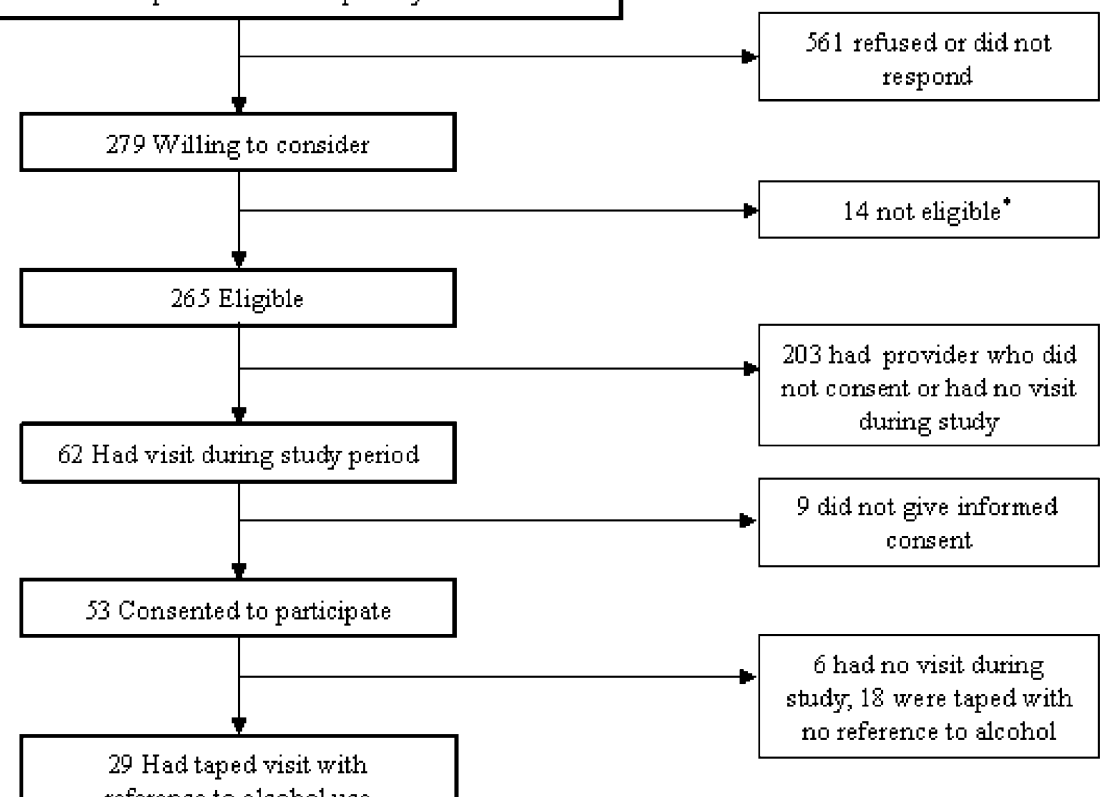

FIGURE 1. Patient recruitment * 4 women excluded, 9 deceased, 1 no contact information.

while others responded with statements that downplayed the potential significance of their patient's drinking (Table 2; Example 2). Some of these minimizing statements were direct dismissals, as in the case of 1 provider, who responded to his patient's self-reported "boozing" with, "I know. I'm more concerned about your kidney function..." (Table 2; Example 2). Finally, patients often disclosed problematic alcohol use in the context of a discussion about another health-related topic, such as medication adherence or weight. In response, providers frequently pursued the nonalcohol-related issue, despite vivid patient statements indicating recognition of a drinking problem (Table 2; Examples 3, 4). Although some patients attempted to reinitiate an alcohol-related discussion, providers who had not pursued their patient's initial disclosure seldom returned to the topic.

In 7 of the 39 visits analyzed, providers did attempt to explore their patients' alcohol use. However, in the majority of these visits, providers asked only closed questions such as "... . and when do you-when do you have the drinks in the day ... Is it before lunch?” or an adapted standardized screening question such as the CAGE question, "You ever need an eye opener or anything like that?" Despite their questioning, these providers usually did not elicit significant information regarding their patient's alcohol use. Coders did identify a single "outlier" in which the provider pursued the issue of alcohol, gathered information, and negotiated a management plan (Table 2; Atypical Example 1).

Advice Offered to Patients About Alcohol Use Was Typically Vague and/or Tentative in Contrast to Smoking-Related Advice, Which Was More Common and Usually More Clear and Firm. Twenty-three of the 39 tapes contained both alcohol- and smoking-related discussions. In these 23 tapes, advice about smoking was more common and differed markedly from alco-

Table 1. Demographic and Clinical Characteristics of Study Participants

\begin{tabular}{lc}
\hline \hline & $N(\%)$ \\
\hline Provider characteristics $(N=14)$ & \\
Sex & \\
$\quad$ Male & $8(57)$ \\
Female & $6(43)$ \\
Provider type & \\
$\quad$ Resident or Fellow MD & $4(29)$ \\
Faculty/staff MD & $5(36)$ \\
Nurse practitioner & $5(36)$ \\
Patient characteristics $(N=29)$ & \\
Age (y) & \\
25 to 49 & $5(17)$ \\
50 to 64 & $12(41)$ \\
R65 & $12(41)$ \\
Race & \\
White & $24(83)$ \\
African American & $3(10)$ \\
Other & $2(7)$ \\
Marital status & \\
Never married & $2(7)$ \\
Married/widowed & $12(41)$ \\
Separated/divorced & $15(52)$ \\
Education: greater than high school & $14(48)$ \\
Income: $<$ \$20,000/y & $18(62)$ \\
Alcohol screening results & \\
Self-report drinking in the past year & $29(100)$ \\
Self-report $\geq 14$ drinks/wk, past year & $14(48)$ \\
Self-report $\geq 6$ drinks on an occasion, past year & $27(93)$ \\
CAGE score $\geq 1$ ("ever" timeframe) & $17(59)$ \\
Self-report problem drinking, ever & $11(40)$ \\
\hline &
\end{tabular}


Table 2. Examples of Theme 1: Patients Disclosed Information Regarding Their Drinking, But Providers Often Did Not Explore These Disclosures

Example 1

Patient A: "Six beers . . . or maybe even 8 sometimes"

Provider 1: "Okay. Okay. Have you been able to take your medication on a regular basis?"

No further exploration of patient's drinking during this visit

Example 2

Provider 2: "So maybe plain Tylenol would be a better choice ..."

Patient B: ". . I I was worried about my liver on that. You said it's bad for your liver and then-"

Provider 2: "I'm more concerned-"

Patient B: "Well, I've been boozing"

Provider 2: "I know. I'm more concerned about your kidney function ..."

Only reference to alcohol during this visit

Example 3

Provider 3: ". . . you were drinking 4 or 5 drinks a day ..."

Patient C: “. . . I-I backed off of that because you talked about weight"

Provider 3: "Yeah. Yeah. I noticed your weight's down a bit"

(199 lines later...)

Patient C: "I took Nyquil until I passed out and busted my fool head open. I fell off a chair"

Provider 3: "Oh. Well, we didn't check any lab tests at your last visit"

No other reference to patient's drinking during this visit

Example 4

Patient D: "I freaked out and tried to self-medicate with alcohol . . . they got me [to the hospital] . . and sobered me up . . they took my blood sugar and it was up to 400 something . . . so I figured this last time when I was drinking that my blood sugar was probably way out of sight . . . because I drank enough to ... raise my blood sugar way up there ..."

Provider 4: "Well, how have your-have-have you been checking your sugars at all in the last few days?"

No further exploration of patient's drinking during this visit

Atypical example 1

Provider 5: "I had some concerns after our last visit because you said that you tend to have maybe 6 drinks or so, or 3 doubles or something at night with the ..."

Patient E: "No. I have-Well, yeah, I guess you'd call them doubles"

Provider 5: "Mm-hmm"

Patient E: "I have 3 martinis ... And that's a regular routine ... And I-I enjoy it"

Provider 5: "Yeah, I understand that"

..

Provider 5: "Has she or anybody else ever suggested that you stop or slow down drinking?"

Patient E: "Well, you consider that heavy. See, I don't. It's-and ..."

Provider 5: "But anybody else in your life ever . . . ever say any..."

Patient E: "No. No. No. I'm up by myself until . . what? ... 12, 1 o'clock in the morning"

Provider 5: "Mm-hmm"

Patient E: "And I enjoy that time"

...

Provider 5: "There is the issue of how it interacts with your diabetes . . . And the blood sugar. And I think we need to maybe get some-we need to get some more objective data, you know, uh, find out what your sugar does, what effects uh, uh, your ... your..."

Patient E: "Mm-hmm. Well, yeah. I understand it turns into sugar"

Provider 5: “ . . That's the problem with alcohol is that it causes-I mean, it spikes in your blood sugar. And that can be difficult to control even with medication ..."

Patient E: "You're actually right [that my drinking is not good given my diabetes]. But I'm 66 (laughs) and ..."

Provider 5: "Would you be willing today to uh, uh—it's been awhile since we did-you haven't had labs for a long time, actually—to do some labs"

Patient E: "Sure. You bet"

Provider 5: "And we can also look for some markers on-on the alcohol and see if that's uh-if there's objective, uh, physical findings"

Patient E: "Okay. Fine"

Provider 5: "Then it might be a problem"

Patient E: "That sounds good to me"

hol-related advice with regard to tone and content. Advice about smoking and alcohol differed even when offered by the same provider during a single visit (Table 3).

When giving advice about smoking, providers tended to use language that was decisive, clearly stating the course of action that they believed their patient should pursue. Moreover, providers moved beyond merely advising tobacco cessation and gave considerable attention to how patients could accomplish this goal. In contrast, alcohol-related advice was marked with equivocating statements and ambiguity (Table 3). Providers made timid suggestions (e.g., "it'd be worthwhile trying to cut that back a little bit if you can"), and offered vague advice (e.g., "you're going to need to be real careful about that drink- ing. ..”). In addition, most providers did not educate their patients about recommended drinking limits. Direct suggestions regarding how patients might go about changing their drinking behavior were also mostly absent from these alcohol-related discussions.

Very few instances of clear, unambiguous alcohol-related advice were noted. We present the strongest example of such advice in Table 2 (Atypical Example 1).

Discomfort on the Part of the Provider was Evident During Alcohol-Related Discussions. We observed a number of specific behaviors that seemed to indicate discomfort on the part of the provider (Table 4). These included hesitation and stutter- 
Table 3. Examples of Theme 2: Advice About Alcohol Use was Typically Vague and/or Tentative, Whereas Smoking-Related Advice was More Common and Usually More Clear and Firm

Alcohol-related advice

*Provider 1: “. . . we don't ever really recommend that you drink.” ... “[Four to six drinks per day] is a little excessive.”. . “. . it'd be worthwhile trying to cut that back a little bit if you can" (Patient F)

*Provider 4: “ . . y you're going to need to be real careful about that drinking . . . you sound like you get yourself into trouble when you [laughs] get too much on board ..." (Patient D)

Provider 2: "Probably the best, uh, the most important thing is for you to stay sober and that's great you're able to stop and uh, you know, it's-it's hard to do. So apparently, if you have a good support system and stick with some aftercare or else the, the outpatient program here even though you stopped I think it's worthwhile to keep on with that" (Patient A)

Provider 5: "Right. If you've developed that pattern over a long period of time and, you know, I guess, um, when I look at-you know, statistically, um, looking at, um, a drinking pattern that involves, if we say three of those kind of drinks, um, a night, um, 21 over the course of a week, if that's your pattern, that statistically puts you in a category to be-to have-to have more problems, uh, um, social problems, um, health problems, those kind of things, um ..." (Patient E)

Smoking-related advice

*Provider 1: "The important things are to sort of set a specific date when you're going to quit. Because it's easy to say, yeah, I'm going to quit some day. But what you need to do is say, I'm going to quit next-in, you know, 2 or 3 weeks, and let people around you know that, yeah, this is when I'm going to stop smoking so they aren't tempting you, and they can help you. . . . "I'm going to support you when you're, you know, when you're feeling that craving and try to help you get through the-the worst part of it" ... "And give some real thought to quitting smoking" (Patient F)

*Provider 4: "[Your wife] should quit too. . .you need to bring your whole household in" .. "I'd like to see you have a little bit more of a plan in place." “. . .I want you to go [to a smoking cessation clinic]" (Patient D)

Provider 1: "And the smoking can affect your blood pressure too ..." (Patient A)

Provider 6: " . . . I would encourage you to keep working on the smoking cessation" (Patient G)

Provider 6: "I strongly believe that the smoking is worsening your health" (Patient J)

Provider 7: “. . . I want to shake on it that you'll stay off them [cigarettes] or if you feel the urge you give me a call” (Patient H)

*Advice about alcohol and tobacco use offered during the same visit.

ing, inappropriate laughter, and ambiguous statements. These behaviors were not observed or were less apparent when alcohol was not being discussed. With the exception of extremely brief discussions (e.g., a few sentences), all taped alcohol-related encounters included 1 or more of these behaviors.

\section{DISCUSSION}

Three themes emerged from this single-site audiotape study of alcohol-related discussions between patients who screened positive for alcohol misuse and their primary care providers. First, patients disclosed information regarding their drinking, but providers often did not explore these offerings. Second, alcohol counseling was typically vague and tentative in contrast to smoking-related advice, which was more common and usually more clear and firm. Third, provider discomfort was observed in the majority of alcohol-related discussions. These findings suggest that provider discomfort and avoidance may be important barriers to discussions about alcohol use in primary care.

Provider communication skills influence quality of care, ${ }^{37}$ and certain provider behaviors can significantly affect health outcomes such as medication adherence. ${ }^{38}$ Moreover, studies using conversation analysis demonstrate that providers shape the medical encounter in subtle, but important ways. ${ }^{35,36}$ Based on the notion that "conversational utterances are social objects that accomplish actions and activities without necessarily formulating them as such, ${ }^{36}$ conversational analysis suggests that providers' words contain underlying meaning, which patients readily interpret and respond to. In this way, provider communication practices can significantly influence the medical encounter. As this literature suggests, the quality and outcome of alcohol-related discussions are determined, at least in part, by the way in which providers talk to their patients about drinking.

Several studies have directly observed primary care providers' discussions with patients who misuse alcohol, ${ }^{33,39}$ but

\section{Table 4. Examples of Theme 3: Discomfort on the Part of the Provider was Evident During Alcohol-Related Discussions}

Hesitation and stuttering

Provider 1: "But how do you feel about your-your drinking? Do you—do you—do you ..."

Patient A: "What do you mean by that?"

Provider 1: "Do you think like—do you feel like you drink more than your should or do you think it's just a reasonable amount or ...."

Patient A: "Well, I know I do drink more than I should"

Provider 8: "Um, and uh, one of the other things that we had talked about in your follow-up visit is uh, um, is uh, you know, talked about the alcohol and uh, uh ..." (Patient K)

Laughter

Provider 4: “. . you sound like you get yourself into trouble when you [laughs] get too much on board ...” (Patient D)

Patient L: "And I told [my other doctor], you know, is, uh, I have very, uh, poor appetite, and I have a couple beers in the evening. And he says, Well, that's not bad at all. He says, there is some nutrition in that"

Provider 9: [Laughs] "Yeah"

Ambiguous statement

Provider 10: "Well, if you've been successful at reducing your beer intake, good for you. But, you know, you can kind of nurse it along” (Patient M)

Provider 8: "It doesn't sound like it's—it's at the point of being excessively—you're certainly having a lot more at one time" (Patient K) 
no study has qualitatively analyzed these discussions. Patientprovider interactions around other sensitive issues have been examined, and such studies have described behaviors similar to those reported here. Epstein et al. ${ }^{40}$ analyzed videotaped discussions of HIV risk during primary care visits. They found that each encounter contained "awkward moments," and that providers frequently avoided discussions of HIV risk. ${ }^{40}$ Another study, which examined interactions between drug-using inpatients and their providers, found that providers avoided addressing patients' pain and addiction-related issues, lacked a standard approach, and demonstrated discomfort during these interactions. ${ }^{41}$ Finally, Meredith and Marzel $^{42}$ found that providers may lack the skills needed to effectively assess and manage patients with depression. As these studies suggest, societal stigma associated with certain behaviors or conditions often infiltrate the medical encounter and may adversely impact provider communication skills.

A number of studies have reported that providers expect their patients to avoid discussion of alcohol misuse or to deny problem drinking. ${ }^{19,24,28}$ However, patients in this study made extremely frank statements about their alcohol use. We also found that patients frequently disclosed information about their drinking practices, with and without provider prompting. Moreover, as indicated by their initiation and sometimes re-initiation of the alcohol-related discussion, many patients seemed to be seeking information, advice, and/or assistance. These behaviors suggest that patients are willing to engage in dialogue about their drinking with a primary care provider. However, providers in this study frequently avoided discussions of alcohol misuse by abruptly changing the subject, minimizing the patient's statement, or pursuing a nonalcoholrelated issue. Although primary care providers must manage many competing priorities, ${ }^{43}$ and providers in the present study did address other health-related behaviors, our findings of avoidance combined with notable discomfort suggest that providers were not comfortable discussing alcohol use.

The present study also found marked differences between smoking- and alcohol-related discussions. Larsson et al. ${ }^{44}$ analyzed audiotaped discussions about tobacco and alcohol use between 3 specialist providers and 20 patients whose drinking and smoking practices were unknown. They found that alcohol-related discussions were often superficial and yielded little information regarding patients' drinking practices. ${ }^{44}$ Another audiotape study, which examined behavioral counseling strategies in a primary care setting, found that patients tended to dominate discussions of alcohol use. ${ }^{45}$ The authors also noted that providers seemed most in control of discussions about smoking and diet, offering more "how-to" information compared with other preventive care topics. ${ }^{45}$ Finally, a Finnish study, in which providers were interviewed and their medical records sampled, found that smoking was discussed more often than alcohol and that providers perceived smoking to be easier to identify and discuss. ${ }^{23}$

The observed disparities between smoking- and alcoholrelated discussions may reflect several factors. As hypothesized by Russell and Roter, ${ }^{45}$ the broad dissemination of information regarding counseling for tobacco use may have helped providers develop "scripts." In other words, for smoking cessation counseling, providers "know what they want to say and how to say it." ${ }^{45}$ Providers may not have such scripts for alcohol counseling. Advising patients about smoking may also be more straightforward as any smoking is harmful and patients can routinely be advised not to smoke. ${ }^{23}$ In contrast, while excessive alcohol use is clearly hazardous, drinking within recommended limits is socially accepted. ${ }^{46,47}$ Providers may also perceive smoking to be a greater health risk than alcohol use. ${ }^{24}$

There are several important limitations to this study. First, patient participants were older, male veterans at a single clinic, and findings may not extend to other settings. Also, the audiotapes may not have captured nonverbal messages conveyed by patients or providers. No information is available about previous alcohol-related discussions that may have taken place between the participating patient-provider pairs. In addition, although they screened positive for alcohol misuse, some patients may not have been misusing alcohol at the time of their visit. Finally, findings may have been influenced by recruitment bias, as a large number of potentially eligible subjects were not included in the study. It is possible that nonparticipating providers may have been less comfortable than participating providers with their communication skills.

The present study also has unique strengths. In contrast to most studies of alcohol-related discussions, our findings were derived from audiotape data, rather than patient or provider self-report. The open-ended qualitative approach also allowed us to capture aspects of the patient-provider interaction, which the previous quantitative study could not address. All patients were current drinkers who screened positive for alcohol misuse, and almost all reported drinking 6 or more drinks on an occasion in the past year. Finally, participants were blinded to the study's focus on alcohol-related discussions.

Findings from this exploratory study highlight the need for further investigation into primary care alcohol counseling practices in order to identify communication strategies that facilitate or impede effective discussions of alcohol use. Future studies should also identify strategies that help providers to "mend" problematic alcohol-related conversations. ${ }^{40}$ Such research could inform educational efforts with regard to alcohol counseling.

In conclusion, although primary care alcohol counseling can have tremendous benefits for patients and society, ${ }^{1,2}$ this qualitative study suggests that providers may not be prepared to offer such counseling. Providers in this study avoided discussions of alcohol misuse, and exhibited discomfort when such conversations did occur. The marked differences between smoking- and alcohol-related discussions suggest that, given the necessary knowledge and skills, providers can be taught to offer behavior change counseling effectively. If future studies describe similar patterns, efforts to implement brief alcohol counseling in primary care settings may need to include educational programs aimed at increasing provider comfort and effectiveness with this important role.

The Veterans Affairs (VA) Ambulatory Care Quality Improvement Project (ACQUIP) was funded by VA HSR\&D Grants \#SDR96-002 and IIR99-376. Dr. Bradley is an investigator at the VA Puget Sound Health Care System, is currently supported by the National Institute on Alcohol Abuse and Alcoholism (NIAAA \#K23AA00313), and was a Robert Wood Johnson (RWJ) Foundation Generalist Physician Faculty Scholar at the time of this work. The views expressed in this article are those of the authors and do not necessarily represent the views of the Department of Veterans Affairs, the University of Washington, the NIAAA, or the RWJ Foundation. 


\section{REFERENCES}

1. Institute of Medicine. Broadening the Base of Treatment for Alcohol Problems: A Report of the Committee for the Study of Treatment and Rehabilitation for Alcoholism. Washington, DC: National Academy Press; 1990.

2. U.S. Preventive Services Task Force. Screening and behavioral counseling interventions in primary care to reduce alcohol misuse: recommendation statement. Ann Intern Med. 2004;140:554-6.

3. Bertholet N, Daeppen JB, Wietlisbach V, Fleming M, Burnand B. Reduction of alcohol consumption by brief alcohol intervention in primary care: systematic review and meta-analysis. Arch Intern Med. 2005;165: 986-95.

4. Moyer A, Finney JW, Swearingen CE, Vergun P. Brief interventions for alcohol problems: a meta-analytic review of controlled investigations in treatment-seeking and non-treatment-seeking populations. Addiction. 2002;97:279-92

5. Whitlock EP, Polen MR, Green CA, Orleans T, Klein J. Behavioral counseling interventions in primary care to reduce risky/harmful alcohol use by adults: a summary of the evidence for the U.S. Preventive Services Task Force. Ann Intern Med. 2004;140:557-68.

6. Ballesteros J, Duffy JC, Guerejeta I, Arino J, Gonzalez-Pinto A. Efficacy of brief interventions for hazardous drinkers in primary care: systematic review and meta-analyses. Alcohol Clin Exp Res. 2004;28: 608-18.

7. Wallace $\mathbf{P}$, Cutler S, Haines A. Randomised controlled trial of general practitioner intervention in patients with excessive alcohol consumption. BMJ. 1988;297:663-8.

8. Fleming MF, Barry KL, Manwell LB, Johnson $\mathbf{K}$, London R. Brief physician advice for problem alcohol drinkers: a randomized controlled trial in community-based primary care practices. JAMA. 1997;277: 1039-45

9. Fleming MF, Mundt MP, French MT, Manwell LB, Stauffacher EA, Barry KL. Benefit-cost analysis of brief physician advice with problem drinkers in primary care settings. Med Care. 2000;38:7-18.

10. Fleming MF, Mundt MP, French MT, Manwell LB, Stauffacher EA, Barry KL. Brief physician advice for problem drinkers: long-term efficacy and benefit-cost analysis. Alcohol Clin Exp Res. 2002;26:36-43.

11. Samet JH, Rollnick S, Barnes H. Beyond CAGE: a brief clinical approach after detection of substance abuse. Arch Intern Med. 1996;156:2287-93.

12. Barnes HN, Samet JH. Brief interventions with substance-abusing patients. Med Clin North Am. 1997;81:867-79.

13. Rush B, Ellis K, Crowe T, Powell L. How general practitioners view alcohol use: clearing up the confusion. Can Fam Physician. 1994;40: 1570-8.

14. Hasin D, Grant B, Dufour M, Endicott J. Alcohol problems increase while physician attention declines. Arch Internal Med. 1990;150: 397-400.

15. D'Amico EJ, Paddock SMBA, Kung F. Identification of and guidance for problem drinking by general medical providers. Med Care. 2005;43: 229-36.

16. Edlund MJ, Unutzer J, Wells KB. Clinician screening and treatment of alcohol, drug, and mental problems in primary care: results from healthcare for communities. Med Care. 2004;42:1158-66.

17. Babor TE, Higgins-Biddle J, Dauser D, Higgins P, Burleson JA. Alcohol screening and brief intervention in primary care settings: implementation models and predictors. J Stud Alcohol. 2005;66:361-8.

18. Funk M, Wutzke S, Kaner E, et al. A multicountry controlled trial of strategies to promote dissemination and implementation of brief alcohol intervention in primary health care: findings of a World Health Organization collaborative study. J Stud Alcohol. 2005;66:379-88.

19. Arborelius E, Thakker KD. Why is it so difficult for general practitioners to discuss alcohol with patients? Fam Practice. 1995;12:419-22.

20. Lock CA, Kaner E, Lamont S, Bond S. A qualitative study of nurses' attitudes and practices regarding brief alcohol intervention in primary health care. J Adv Nurs. 2002;39:333-42.

21. Beich A, Gannik D, Malterud K. Screening and brief intervention for excessive alcohol use: qualitative interview study of the experiences of general practitioners. BMJ. 2002;325:1-5.

22. Bander KW, Goldman DS, Schwartz MA, Rabinowitz E, English JT. Survey of attitudes among three specialties in a teaching hospital toward alcoholics. J Med Educ. 1987;62:17-24.
23. Aira M, Kauhanen J, Larivaara P, Rautio P. Differences in brief interventions on excessive drinking and smoking by primary care physicians: qualitative study. Prev Med. 2004;38:473-8.

24. Aira M, Kauhanen J, Laricaara $\mathbf{P}$, Rautio $\mathbf{P}$. Factors influencing inquiry about patients' alcohol consumption by primary health care physicians: qualitative semi-structured interview study. Fam Practice. 2003;20:270-5.

25. Aalto M, Pekuri P, Seppa K. Obstacles to carrying out brief intervention for heavy drinkers in primary health care: a focus group study. Drug \& Alc Review. 2003;22:169-73.

26. Anderson $\mathbf{P}$, Kaner E, Wutzke SE, et al. Attitudes and managing alcohol problems in general practice: an interaction analysis based on findings from a WHO collaborative study. Alcohol Alcohol. 2004;39:351-6.

27. Cornuz J, Ghali WA, Di Carlantonio D, Pecoud A, Paccaud F. Physicians' attitudes towards prevention: importance of intervention-specific barriers and physicians' health habits. Fam Pract. 2000; 17:535-40.

28. Thom B, Tellez C. A difficult business: detecting and managing alcohol problems in general practice. Br J Addict. 1986;81:405-18

29. Fihn SD, McDonell MB, Diehr P, et al. Effects of sustained audit/ feedback on self-reported health status of primary care patients. Am J Med. 2004;116:241-8.

30. National Institute on Alcohol Abuse and Alcoholism, U.S. Department of Health and Human Services, National Institute of Health. Helping Patients Who Drink Too Much: A Clinician's Guide. Rockville, MD; 2005.

31. Bradley KA, Kivlahan DR, Bush KR, McDonell MB, Fihn SD. Variations on the CAGE alcohol screening questionnaire: strengths and limitations in VA general medical patients. Alcohol Clin Exp Res. 2001;25: 1472-8.

32. Bradley KA, Bush K, McDonell MB, Malone T, Fihn SD. Screening for problem drinking: comparison of CAGE and AUDIT. J Gen Intern Med. 1998;13:379-88.

33. Bradley KA, Epler A, Bush K, et al. Alcohol-related discussions during general medicine appointments of patients who screen positive for atrisk drinking. J Gen Intern Med. 2002;17:315-27.

34. Crabtree BF, Miller WL, eds. Doing Qualitative Research. 2nd ed. Thousand Oaks, CA: Sage Publications Inc; 1999.

35. Drew P, Chatwin J, Collins S. Conversation analysis: a method for research into interactions between patients and health-care professionals. Health Expect. 2001;4:58-70.

36. Maynard DW, Heritage J. Conversation analysis, doctor-patient interaction and medical communication. Med Educ. 2005;39:428-35.

37. Roter DL, Frankel RM, Hall JA, Sluyter D. The expression of emotion through nonverbal behavior in medical visits. mechanisms and outcomes. J Gen Intern Med. 2006;21(Suppl 1):S28-S34.

38. Beck RS, Daughtridge R, Sloane PD. Physician-patient communication in the primary care office: a systematic review. J Am Board Fam Pract. 2002;15:25-38.

39. Wenrich MD, Paauw DS, Carline JD, Curtis JR, Ramsey PG. Do primary care physicians screen patients about alcohol intake using the CAGE questions? J Gen Intern Med. 1995;10:631-4.

40. Epstein RM, Morse DS, Frankel RM, Frarey L, Anderson K, Beckman HB. Awkward moments in patient-physician communication about HIV risk. Ann Intern Med. 1998;128:435-42.

41. Merrill JO, Rhodes LA, Deyo RA, Marlatt GA, Bradley KA. Mutual mistrust in the medical care of drug users: the keys to the "Narc cabinet." J Gen Intern Med. 2002;17:327-33.

42. Meredith LS, Mazel RM. Counseling for depression by primary care providers. Int J Psychiatry Med. 2000;30:343-65.

43. Yarnall KSH, Pollak KI, Ostbye T, Krause KM, Micchener JL. Primary care: is there enough time for prevention? Am J Public Health. 2003;93: 635-41.

44. Larsson US, Saljo R, Aronsson K. Patient-doctor communication on smoking and drinking: lifestyles in medical consultations. Soc Sci Med. 1987;25:1129-37.

45. Russell NK, Roter DL. Health promotion counseling of chronic-disease patients during primary care visits. Am J Public Health. 1993;83: 979-82.

46. Green CA, Perrin NA, Polen MR. Gender differences in the relationships between multiple measures of alcohol consumption and physical and mental health. Alcohol Clin Exp Res. 2004;28:754-64.

47. Klatsky AL, Armstrong MA, Friedman GD. Alcohol and mortality. Ann Intern Med. 1992;117:646-54. 